\title{
A Critical Test for Photoionization of Seyfert NLRs
}

\author{
S. K. Metz, ${ }^{1}$ C. N. Tadhunter, ${ }^{1}$ A. Robinson, ${ }^{2}$ and D. J. Axon ${ }^{3}$ \\ ${ }^{1}$ Dept. of Physics, University of Sheffield, S3 7RH, UK \\ ${ }^{2}$ Department of Physical Sciences, University of Hertfordshire, Hatfield, \\ Hertfordshire AL10 9AB, UK \\ ${ }^{3}$ S[Space Telescope Science Institute, Baltimore, MD 21218, USA
}

\begin{abstract}
We present measurements of the central ionizing photon flux $q$ across three spatially resolved Seyfert NLRs, with implications for the ionization mechanisms.
\end{abstract}

\section{A critical test with a surprising result}

A common assumption of models of Seyfert NLRs is that the line-emitting gas is photoionized by photons emitted by a central source. There is evidence to support this assumption for the ENLR, but detailed observations of the NLR (high [O III] temperatures; disturbed kinematics; close association with radio structures) are driving the evolution of more complex models involving shocks (see Morse et al. 1996) or a two-component ionizing continuum (Binette et al. 1996).

A critical test of the central source model uses the ionization parameter, defined as the ratio of the density of ionizing photons to gas density, which can be written as

$$
U=\frac{q}{n_{e} r^{2} c}>\frac{q}{n_{e}(r / \cos \theta)^{2} c}
$$

where $q$ is the number of ionizing photons emitted per unit time per steradian, $n_{e}$ is the electron density, $r$ is the distance of the emission-line cloud from the source, and $\theta$ is the angle between a line from the source to the cloud and the plane of the sky. Using $[\mathrm{O} \mathrm{III}] \lambda 5007 /[\mathrm{O} \mathrm{II}] \lambda 3727$ to determine $U$, $[\mathrm{S} \mathrm{II}] \lambda 6717 / \lambda 6731$ for the density, and estimating $r$, allows a straightforward determination of $q$. If the central source assumption is correct, wherever we measure $q$, we should get the same value.

Using data obtained with long-slit spectroscopy at the WHT on La Palma, we measured $q$ across the NLR of three nearby Seyferts - NGC 4151, NGC 2110, and NGC 2992 - with the slit aligned along the radio structure. As Fig. 1 shows, far from being constant as expected, $q$ rises dramatically with increasing distance from the nucleus.

To explain this result in terms of a projection effect requires the projection factor to increase, i.e., $\theta$ to increase, towards the nucleus in all three objects. This rules out the sharp-edged geometry of a filled bi-cone. This would also mean the value of $q$ derived at the boundary of the NLR is closest to the true 


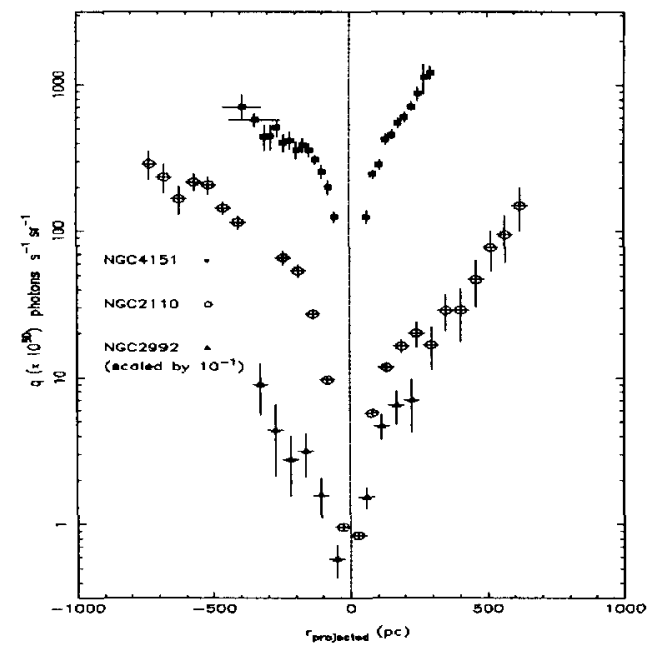

Figure 1. Variation of $q$, the ionizing photon flux, with radius in NGC 4151, NGC 2110, and NGC 2992. If all the photons come from the central source, then $q$ should be constant $\left(H_{0}=75 \mathrm{~km} \mathrm{~s}^{-1} \mathrm{Mpc}^{-1}\right)$.

value. Fixing $q$ at this value allows an estimate of the de-projected $r$, e.g., for NGC 2110, setting $q=100 \times 10^{50}$ photons s $\mathrm{s}^{-1} \mathrm{sr}^{-1}$ implies a minimum distance of the clouds from the nucleus of $240 \mathrm{pc}$, placing a strong constraint on the NLR geometry.

A two-component ionizing continuum due to a mixture of ionization-bounded and matter-bounded clouds cannot account for an increase in $q$ by factors of $10^{2}-10^{3}$.

The extended radio sources coincident with the NLR, suggest that highvelocity shocks due to interaction of the radio jet with the gas clouds could be a source of ionizing photons. Alternatively, shock ionization, producing significantly lower $\left[\mathrm{O}_{\mathrm{III}}\right] \lambda 5007 /\left[\mathrm{O}_{\mathrm{II}}\right] \lambda 3727$, could become more important with decreasing $r$, and would in fact have to dominate close to the nucleus.

\section{Conclusion}

The dramatic increase in $q$ with increasing projected $r$ in the NLRs of these objects places unfeasible constraints on the geometry of one component central source photoionization models, and poses serious problems for the distribution and make-up of of the clouds in the ionization-bounded/matter-bounded cloud mix model. This supports the inclusion of jet-induced shocks, although a consistent model remains elusive.

\section{References}

Binette, L., Wilson, A.S., \& Storchi-Bergmann, T. 1996, A\&A, 312, 365.

Morse, J. A., Raymond, J. C., \& Wilson, A. S. 1996, PASP, 108, 426. 